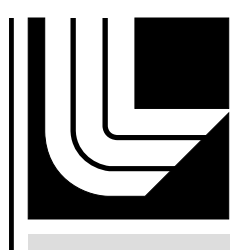

LAW RENCE LIVERMORE N A T IO N A L LABORATORY

On the Initiation of High

Explosives by Laser Radiation

A. M. Rubenchik

March 29, 2006

Propellants, Explosive, Pyrotechnices 
This document was prepared as an account of work sponsored by an agency of the United States Government. Neither the United States Government nor the University of California nor any of their employees, makes any warranty, express or implied, or assumes any legal liability or responsibility for the accuracy, completeness, or usefulness of any information, apparatus, product, or process disclosed, or represents that its use would not infringe privately owned rights. Reference herein to any specific commercial product, process, or service by trade name, trademark, manufacturer, or otherwise, does not necessarily constitute or imply its endorsement, recommendation, or favoring by the United States Government or the University of California. The views and opinions of authors expressed herein do not necessarily state or reflect those of the United States Government or the University of California, and shall not be used for advertising or product endorsement purposes. 


\title{
On the Initiation of High Explosives by Laser Radiation
}

\author{
A. M. Rubenchik \\ University of California \\ Lawrence Livermore National Laboratory \\ Livermore, CA 94551
}

The problem of laser initiation of high explosives in munitions is considered. In this situation, the laser illuminates a small spot on the casing, and lateral thermal transport affects the initiation temperature. We use a variational method to calculate the critical temperature for explosive initiation as a function the laser spot size, for common high explosives. The effect of the dwelling time of the irradiation is then evaluated. We demonstrate that in typical situations the critical temperature is determined by the dwelling time rather than by the laser spot size.

Keywords: High explosive, laser initiation, TNT, heat transport, thermal explosion.

\section{Introduction}

When a laser beam heats a munition, the high temperature produced by the laser can ignite the high explosive (HE) and destroy the munition, even before the beam penetrates the metal casing. Below we will estimate the temperature required to initiate the ignition.

When the explosive is heated, the internal temperature $T$ is described by the heat conduction equation with a source term produced by the chemical reactions [1-5]:

$$
\rho C \frac{\partial T}{\partial t}=\kappa \nabla^{2} T+\rho Q Z e^{-\frac{E}{T}}
$$

Here $\kappa$ is the thermal conductivity of the HE, $\rho$ the density, $C$ the heat capacity, $Q$ the heat of decomposition, $E$ the activation energy, and $Z$ the pre-exponential factor. For the common high explosives RDX and TNT, the values for these parameters are listed in Table 1. 


\begin{tabular}{|l|c|c|c|c|c|c|}
\hline & $\rho, \mathrm{g} / \mathrm{cm}^{3}$ & $C, \mathrm{~J} /(\mathrm{g} \mathrm{K})$ & $\kappa, \mathrm{W} /(\mathrm{K} \mathrm{cm})$ & $Q, \mathrm{~kJ} / \mathrm{g}$ & $Z, 1 / \mathrm{s}$ & $E, \mathrm{eV}$ \\
\hline RDX & 1.8 & 2.1 & 0.003 & 2.1 & $10^{18.5}$ & 2.07 \\
\hline TNT & 1.6 & 2.1 & 0.00215 & 2.1 & $10^{12.2}$ & 1.89 \\
\hline
\end{tabular}

Table 1. Parameters for RDX and TNT, from [3].

The initiation of HE surrounded by walls with fixed temperature $T$ is a wellknown problem [1-3]. Below some critical temperature $T_{c}$, the volume heat production is compensated by the thermal flux through the walls. When the temperature becomes sufficiently high, thermal transport is unable to compensate for the energy production by chemical reactions, and a thermal explosion takes place. The critical temperature can be found as a maximal boundary temperature for which a stationary solution exists [1-3]. It is given by the solution of the equation

$$
\frac{E}{T_{c}}=\ln \left(\frac{a^{2} \rho Q Z E}{\delta \kappa T_{c}^{2}}\right)
$$

Here $a$ is the radius of the HE volume and $\delta$ is a geometrical factor. The latter parameter is 3.32 for a sphere, 2.0 for an infinite cylinder, and 0.88 for an infinite slab.

In typical cases, we have $E>>T_{c}$. Then with logarithmic accuracy, we can solve Eq. (1.2) explicitly for the critical temperature, obtaining

$$
\frac{E}{T_{c}}=\ln \left[\frac{a^{2} \rho Q Z}{\delta \kappa E} \ln ^{2}\left(\frac{a^{2} \rho Q Z}{\delta \kappa E}\right)\right]
$$

Naturally, the critical temperature is minimal for a spherical volume and maximal for a slab, but the numerical difference is not large due to the weak dependence on the geometrical factor. Figure1 shows the critical temperatures of two types of HE, as calculated with Eq. (1.3) in cylindrical geometry. 


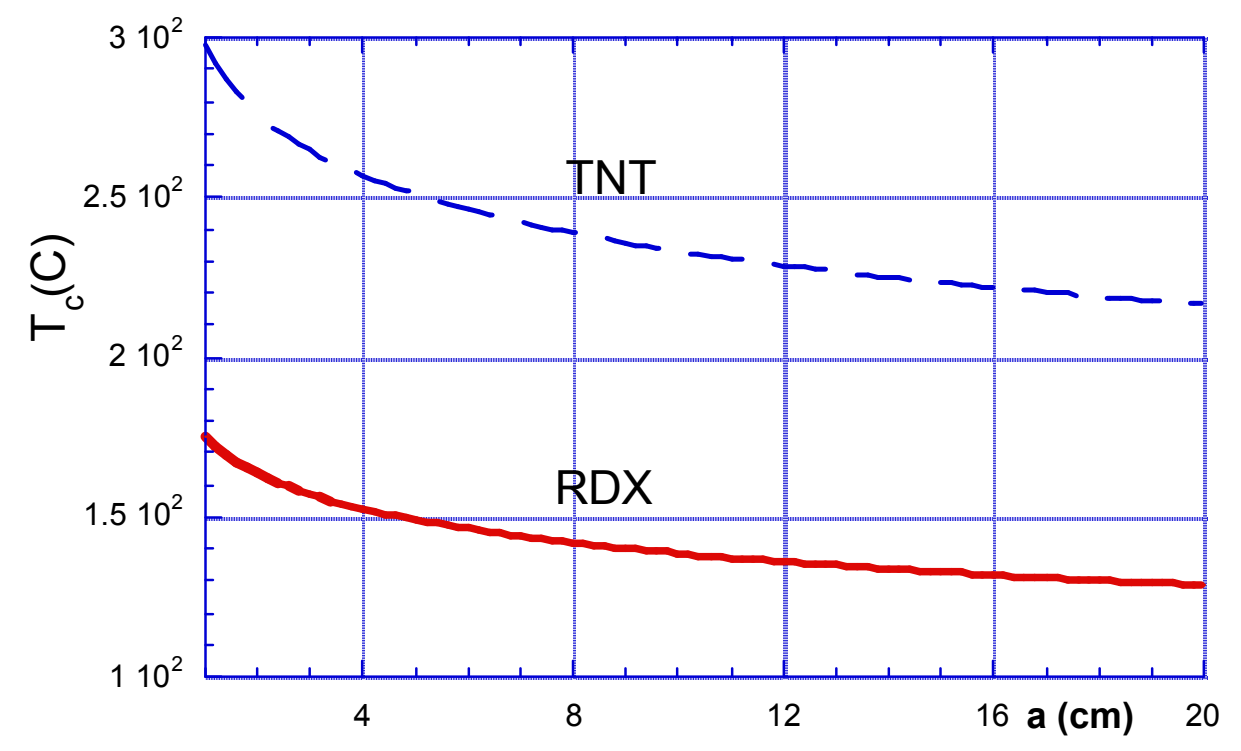

Fig. 1. Critical temperatures for cylindrical samples of RDX and TNT.

In the case of a laser-initiated explosion we have a different boundary condition, namely a hot spot with radius $a$ on the boundary. The critical temperature will be determined as the temperature at which the steady state solution of (1.1) with proper boundary conditions exists.

\section{Critical Temperature for Laser Initiation}

We must solve the steady state version of Eq. (1.1):

$$
\nabla^{2} T+\frac{\rho Q Z}{\kappa} e^{-\frac{E}{T}}=0 .
$$

The boundary condition on the metal/HE interface $(z=0)$ is

$$
T(r, z=0) \equiv T_{0}(r)=T_{0} \exp \left(-r^{2} / a^{2}\right) .
$$


We assume for simplicity a Gaussian temperature distribution on the spot. Since the thermal conductivity of the metal is very high in comparison with that of HE, within the metal we can ignore temperature gradients. From the conservation of thermal flux we have another boundary condition for Eq. (2.1):

$$
\frac{\partial T}{\partial z}=0
$$

at $\mathrm{z}=0$. We can eliminate Eq. (2.3) by considering instead a semi-space solution in all space, symmetric with respect to the plane $z=0$.

Following Frank-Kamenetskii [1], we will approximate the Arrhenius exponential function as a power law via

$$
e^{-\frac{E}{T}} \approx c T^{m}
$$

This approximation is sufficiently good over a given portion of the temperature interval.

The constants $c$ and $m$ are found from the condition that both the functions and their derivatives are equal at $T=T_{0}$. We have

$$
e^{-\frac{E}{T}} \approx e^{-\frac{E}{T_{0}}}\left(\frac{T}{T_{0}}\right)^{\frac{E}{T_{0}}}
$$

The equation for the dimensionless temperature $u=T / T_{0}$ has the form

where

$$
\nabla^{2} u+B u^{m}=0
$$

$$
m=E / T_{0}, \quad B=e^{-m} \frac{\rho Q Z}{\kappa T_{0}} .
$$

Equation (2.4) can be derived from a variational principle, in that it realizes the extrema of the functional

$$
H=\int\left[(\nabla u)^{2}-\frac{2 B}{m+1} u^{m+1}\right] d z r d r
$$


The variational principle opens up the possibility of finding an approximate solution of (2.1). The accuracy of the method is difficult to estimate. In studies of nonlinear self-focusing, however, the method gives results of high accuracy $[6,7]$.

Consider the trial function

$$
u=g(z) e^{-\frac{r^{2}}{a^{2}}} .
$$

Using this trial function and integrating Eq. (2.5) over $r$ we obtain

$$
H=\int d z\left[\frac{a^{2}}{2}(\nabla g)^{2}+g^{2}-\frac{2 B a^{2}}{(m+1)^{2}} g^{m+1}\right] .
$$

The equation for the function $\mathrm{g}$ that realizes the extrema of $H$ is given by

$$
\frac{d^{2} g}{d z^{2}}+\frac{2 B}{(m+1)} g^{m}-\frac{2 g}{a^{2}}=0 .
$$

We are looking for the even solution of this equation subject to the condition $g=1$ at $z=0$. Equation (2.8) is similar to the equation describing the motion of a particle in a potential well, with the potential

$$
U(g)=\frac{2 B}{(m+1)^{2}} g^{m+1}-\frac{g^{2}}{a^{2}} .
$$

We are looking for a localized solution, which in terms the "potential well" description corresponds to zero energy. The solution has a soliton-like shape with maximum at $\mathrm{z}=0$. The maximum of the solution is determined by the condition $U(g)=0$. On the other hand, at this point we have $\mathrm{g}=1$. Therefore the criterion for a solution to exist is

$$
B=\frac{(m+1)^{2}}{2 a^{2}},
$$


which determines the critical temperature for ignition. Returning to the natural variables and using $m=E / T_{c}>>1$, we have

$$
\frac{E}{T_{c}}=\ln \left[\frac{2 \rho Q Z a^{2} T_{c}}{\kappa E^{2}}\right] \approx \ln \left[2 \rho Q Z a^{2} / \kappa E \ln \left[\frac{2 \rho Q Z a^{2}}{\kappa E}\right]\right] .
$$

One can see that the ignition temperature we thereby obtain is higher than that of (1.2), for which the HE is completely surrounded by a heated wall. This result is expected due to the larger role of thermal conduction in the present case.

In Fig. 2 we show the ignition temperatures for RDX and TNT as a function of the laser spot diameter. The ignition temperatures for laser initiation are noticeably higher than for thermal initiation.

\section{Nonstationary effects}

In practice, a laser heats the target for only a short time. As a result, the required initiation temperature increases relative to the quasi-stationary situation treated above. In this situation, the ignition time can be much longer than the laser dwelling time. To ignite the reaction one must not only spend energy to heat the HE up to the ignition temperature but also overcome the heat transport problem. To take the heat capacity into account, we will use a crude approximation as employed, for example, in the theory of gas discharges. We will replace the time derivative in Eq. (1.1) by a term of the form $\mathrm{T} / \tau$. This model is reasonable because in typical situations we deal with a monotonic temperature increase characterized by a single temporal scale $\tau$. Instead of (1.1) we then have

$$
\frac{\kappa T}{a_{\tau}^{2}}=\kappa \nabla^{2} T+\rho Q Z e^{-\frac{E}{T}}
$$




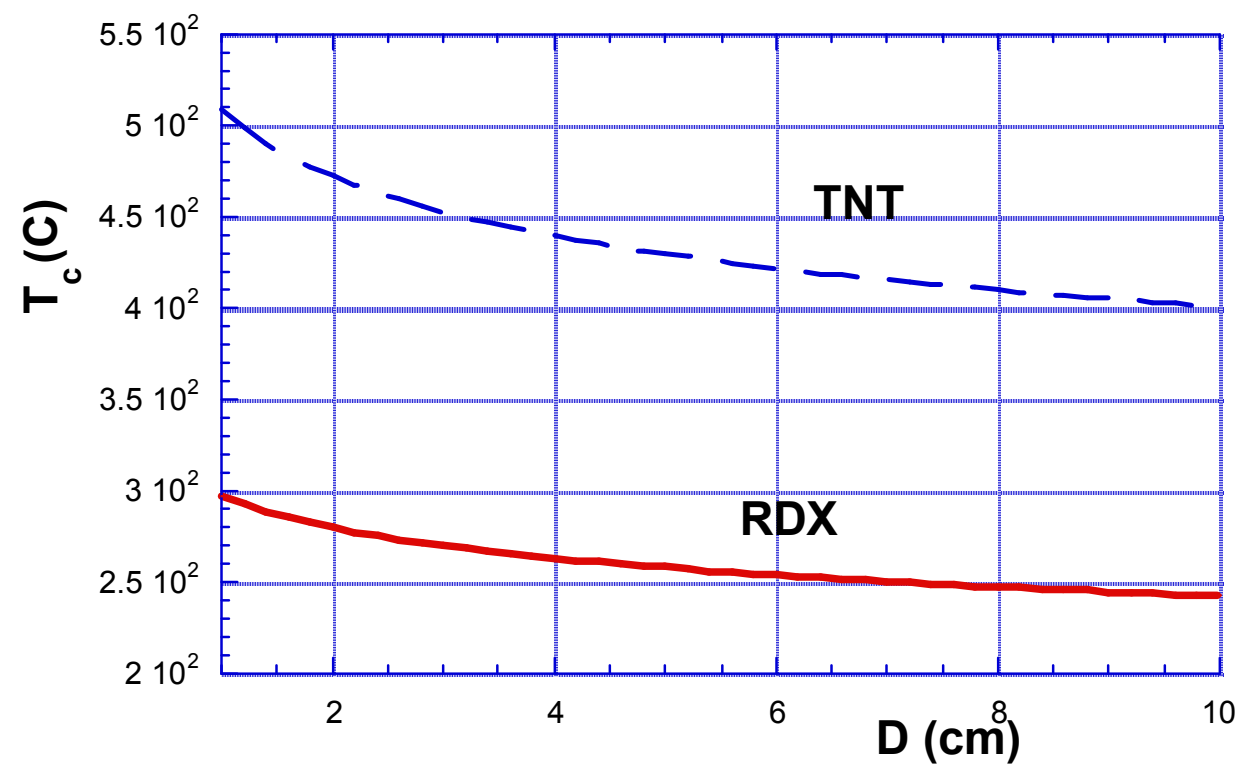

Fig. 2. Ignition temperature for RDX and TNT samples heated by a laser spot with diameter D.

where $a_{\tau}^{2}=\kappa \tau / \rho C$.

This equation must be solved with the boundary conditions (2.2) and (2.3). As we found with Eq. (2.1), the Eq. (3.1) can be derived from a variational principle, which in this case realizes the extrema of the function

$$
H_{\tau}=\int\left[(\nabla u)^{2}-\frac{2 B}{m+1} u^{m+1}+\frac{u^{2}}{a_{\tau}^{2}}\right] d z r d r
$$

Using the same trial functions as in the previous section, we obtain instead of (2.7):

$$
H_{\tau}=a^{2} \int d z\left[\frac{1}{2}(\nabla g)^{2}+\frac{1}{a^{2}} g^{2}+\frac{1}{2 a_{T}^{2}} g^{2}-\frac{2 B}{(m+1)^{2}} g^{m+1}\right]
$$


The subsequent calculations are similar to those presented above. We see that the nonstationary effects can be described by the formula (2.11), provided that the spot radius $a$ is replaced by the effective spot radius $a_{\text {eff, }}$, defined by

$$
\frac{1}{a_{e f f}^{2}}=\frac{1}{a^{2}}+\frac{1}{2 a_{\tau}^{2}}=\frac{1}{a^{2}}+\frac{1}{2 D \tau},
$$

where $D=\kappa / \rho C$ is the thermal diffusivity of the HE.

One can see that nonstationary effects effectively decrease the spot size and increase the ignition temperature. For situations when the laser heats a metal casing which shields the HE, the time $\tau$ is determined by the heat transfer through the metal and can be estimated as follows [8]:

$$
\tau=\frac{\rho_{m} c_{m} T_{c} h}{A I}+\frac{h^{2}}{6 D_{m}} .
$$

Here $h$ is the casing thickness and the thermal parameters pertain to the casing material. Also $A$ is the absorption coefficient and $I$ is the average laser intensity. For steel, the absorptivity is about 0.5 , and the critical temperature is about $650 \mathrm{C}$. The thermal diffusion time becomes

$$
\tau \approx \frac{6.6 h}{I}+0.7 h^{2}
$$

where $h$ is the case thickness in $\mathrm{cm}$ and $I$ is the average intensity in units of $\mathrm{kW} / \mathrm{cm}^{2}$.

If the actual dwelling time is greater than (3.5), then it must be used in (3.4) as $\tau$.

In typical situations, the thermal diffusion scale in a metal casing is comparable to the spot size. The diffusivity of HE is about two orders of magnitude smaller the diffusivity of the metal, so that $a_{\text {eff }} \sim(2 D \tau)^{1 / 2}$. The critical temperature in this situation is determined by the expression 


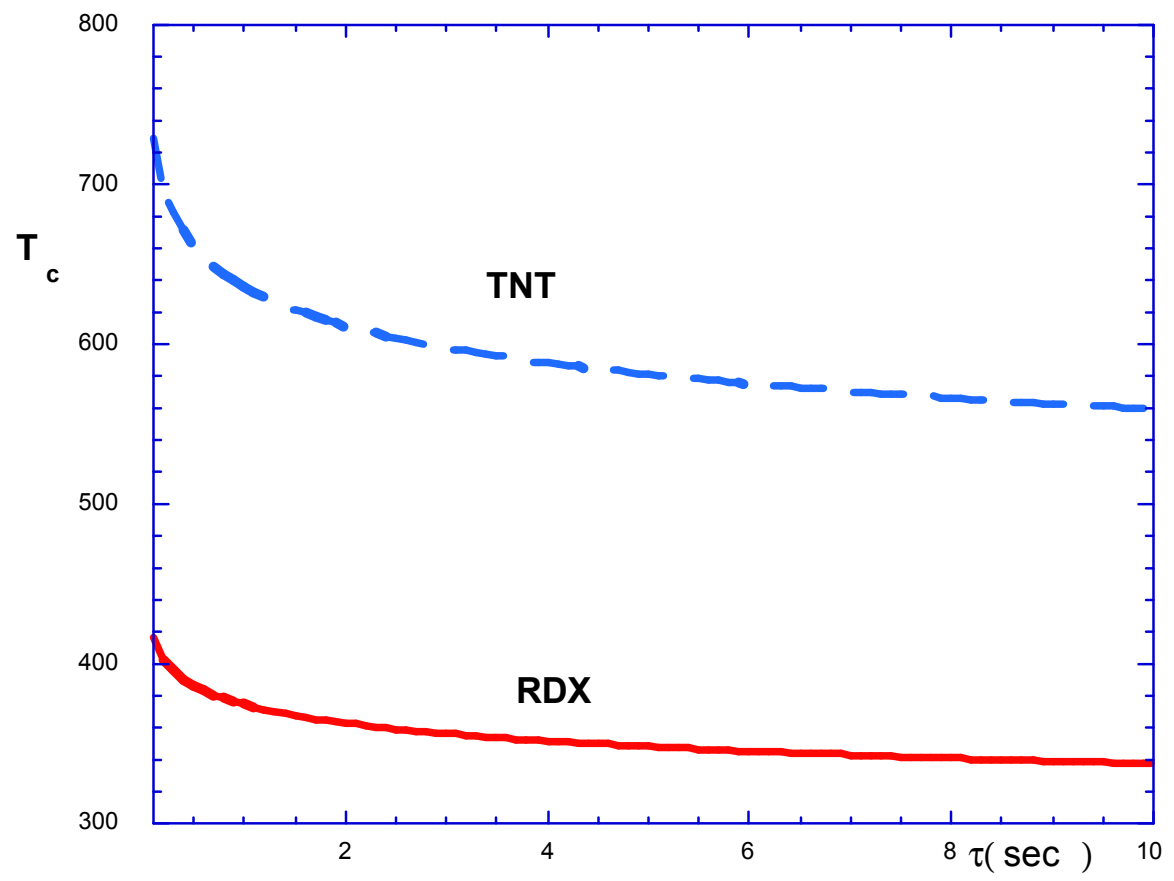

Fig. 3. Ignition temperature for RDX and TNT samples heated by a laser spot with temperature rise time $\tau$.

$$
\frac{E}{T_{c}}=\ln \left[\frac{4 \rho Q Z D \tau T_{c}}{\kappa E^{2}}\right] \approx \ln \left[\rho Q Z D \tau / \kappa E \ln \left[\frac{\rho Q Z D \tau}{\kappa E}\right]\right]
$$

The calculated values of $T_{c}$ for TNT and RDX are shown in Fig.3.

In Ref. [8] the initiation of mortars by laser irradiation was modeled. The radiation was absorbed on the case surface, the thermal flux was transported through the iron case, and the explosive was described by Eq. (1.1). The results are consistent with the results of this paper. For an initiation time of a few seconds, the initiation temperature 
of TNT was about $630 \mathrm{C}$ and was insensitive to the spot size. This temperature is close to $T_{c} \sim 600 \mathrm{C}$, as predicted by our approximate calculations (Fig.3).

\section{Acknowledgments}

This work was supported by the U.S. Army Space and Missile Defense

Command. Work performed under the auspices of the U.S. Department of Energy by the University of California, Lawrence Livermore National Laboratory, under Contract No. W-7405-ENG-48.

\section{References}

[1] D. A. Frank-Kamenetskii, Acta Physiochem. USSR 10, 363 (1939).

[2] L. D. Landau and E. M. Lifshitz, Fluid Mechanics, Pergamon Press, 1959.

[3] J. Zinn and C. L. Mader, J. Appl. Phys. 31, 323-328 (1960).

[4] R. Rogers, Thermochimica Acta 11, 131 (1975).

[5] C. Tarver, R. McGuire, E. Lee, E. Wrenn, and K. Brein, Proc.17th Combustion Symp., 1978.

[6] E. A. Kuznetsov, A. M. Rubenchik, V. E. Zakharov, Phys. Rep. 142, 105 (1986).

[7] V. G. Makhankov, Phys. Rep. 35, 1 (1978).

[8] C. D. Boley and A. M. Rubenchik, “ Simulations of Target Interactions with Pulsed High-Energy Solid State Lasers," Proc. 17th Annual Solid State and Diode Laser Technology Review, Albuquerque, NM, June 8-10, 2004, UCRL-CONF-204087. 